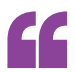

Our study

is the largest RNA

biomarker study for stroke

\title{
STROKE
}

\section{MCEMP1 - a new prognostic and diagnostic biomarker for stroke?}

Levels of MCEMP1 RNA in the peripheral blood could provide a prognostic - and possibly even diagnostic - biomarker for stroke, according to a gene expression profiling study recently reported in Stroke. The MCEMP1 gene encodes mast cell-expressed membrane protein 1 , and the findings add to growing evidence that mast cells have a key role in stroke pathogenesis.

The new research was led by Guillaume Paré at McMaster University in Hamilton, Canada, and was conducted under the auspices of a large case-control study known as INTERSTROKE. "Our study sought to identify peripheral blood RNA biomarkers that distinguish stroke cases from controls and ischaemia from haemorrhage, and predict prognosis," explains lead author Kripa Raman.

The discovery cohort for the new study consisted of 129 individuals with first-time stroke, all of whom had undergone blood sampling within 5 days of symptom onset, and 170 controls with no history of stroke. The results were validated in an independent cohort of 28 stroke cases and 34 controls.

Of the 11,181 genes screened by Raman and colleagues, MCEMP1 showed the most robust association with stroke. In the discovery cohort, the researchers found that, on average, whole-blood MCEMP1 expression was 2.4-fold higher in patients with stroke than in controls. The difference seemed to be even more marked in blood samples collected within $24 \mathrm{~h}$ of stroke onset, although the data were limited.

Expression levels of MCEMP1 correlated with disability at baseline, and with disability and mortality 1 month after stroke, as measured by the modified Rankin Scale. Importantly, addition of this biomarker was found to improve the performance of existing prognostic models.

Among the stroke cases in the discovery cohort, MCEMP1 expression was 2.1-fold higher in participants with intracerebral haemorrhage than in those with ischaemic stroke, suggesting that this biomarker has diagnostic as well as prognostic value. However, the researchers were unable to fully ascertain the diagnostic specificity of MCEMP1, as the study cohorts did not include any individuals with conditions that mimicked stroke.

"Our study is the largest RNA biomarker study for stroke, with four times more participants than previous studies, and is the first to associate gene expression with stroke outcome," concludes Raman. "The results also

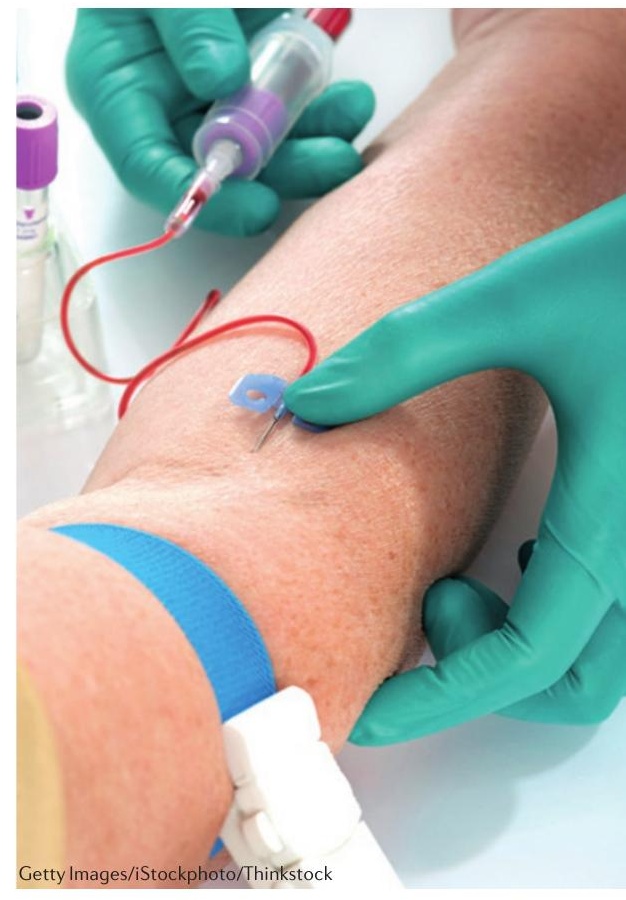

suggest an important role for mast cells in stroke, and further study may lead to the identification of new therapeutic targets."

Heather Wood

ORIGINAL ARTICLE Raman, K. et al. Peripheral blood MCEMP1 gene expression as a biomarker for stroke prognosis. Stroke http//dx.doi.org/10.1161/ STROKEAHA.115.011854 FURTHER READING O'Donnell, M. et al. Rationale and design of INTERSTROKE: a global case-control study of risk factors for stroke. Neuroepidemiology 35, 36-44 (2010) 